\title{
High quality and quantity Genome-wide germline genotypes from FFPE normal tissue
}

Lisa A Cannon-Albright ${ }^{1,2^{*}}$, Kendal G Cooper ${ }^{3}$, Ann Georgelas ${ }^{3}$ and Philip S Bernard ${ }^{4,5}$

\begin{abstract}
Background: Although collections of formalin fixed paraffin embedded (FFPE) samples exist, sometimes representing decades of stored samples, they have not typically been utilized to their full potential. Normal tissue from such samples would be extremely valuable for generation of genotype data for individuals who cannot otherwise provide a DNA sample.

Findings: We extracted DNA from normal tissue identified in FFPE tissue blocks from prostate surgery and obtained complete genome wide genotype data for over 500,000 SNP markers for these samples, and for DNA extracted from whole blood for 2 of the cases, for comparison.

Four of the five FFPE samples of varying age and amount of tissue had identifiable normal tissue. We obtained good quality genotype data for between 89 and $99 \%$ of all SNP markers for the 4 samples from FFPE. Concordance rates of over 99\% were observed for the 2 samples with DNA from both FFPE and from whole blood.
\end{abstract}

Conclusions: DNA extracted from normal FFPE tissue provides excellent quality and quantity genome-wide genotyping data representing germline DNA, sufficient for both linkage and association analyses. This allows genetic analysis of informative individuals who are no longer available for sampling in genetic studies.

\section{Findings}

Our interest in exploring a wider utility for stored FFPE tissue began with the desire for genome-wide (GW) genotyping data for individuals who would be informative to a linkage study in a high-risk pedigree, but who were not available for sampling. Recognizing that normal tissue is typically included in FFPE samples (in addition to tumor tissue), we hypothesized that DNA could be extracted from normal tissue found in most FFPE samples, and that this would provide adequate genome-wide germline DNA for genotyping.

It is recognized that the processes of fixation and storage of FFPE samples lead to degradation and base modification with chemical residues. FFPE-extracted DNA (for tumor tissue) has been reported to be adequate for sequencing, directed genotyping and RNA extraction, but it has been reported that only partial data are typically obtained for genome-wide genotyping. Available genotyping platforms report poor performance

\footnotetext{
* Correspondence: lisa.albright@utah.edu

'Genetic Epidemiology, Department of Internal Medicine, University of Utah School of Medicine, Salt Lake City, UT, USA

Full list of author information is available at the end of the article
}

of GW genotyping data for DNA extracted from FFPE tumor samples; and we could not find published evidence for genome-wide genotyping results for DNA extracted from FFPE normal tissue samples.

Even poor genotyping yields for DNA extracted from FFPE would provide adequate data for genetic linkage studies. Many fewer markers are necessary for genomewide linkage analysis than for genome-wide association analysis (GWAS). For linkage analysis, markers in linkage disequilibrium (LD) are not used (due to introduction of bias), and a much smaller subset of SNP markers can represent the genome. Illumina's original genome-wide SNP set for linkage consisted of 6,000 SNPs. We have previously performed successful genome-wide linkage using subsets of SNPs with no LD from the Illumina 550k SNP data set. We selected 27,157 markers representing the entire genome; median heterozygosity was 0.49 and median spacing was $0.14 \mathrm{cM}[1]$.

\section{Methods}

We identified 5 prostate cancer cases with FFPE tissue samples stored between 18-41 years ago in different facilities (Table 1). A 5-micron cut was made from each
Ciomed Central

(c) 2011 Cannon-Albright et al; licensee BioMed Central Ltd. This is an open access article distributed under the terms of the Creative Commons Attribution License (http://creativecommons.org/licenses/by/2.0), which permits unrestricted use, distribution, and reproduction in any medium, provided the original work is properly cited. 
Table 1 Summary of the 5 tissue block samples tested

\begin{tabular}{lcccl}
\hline $\begin{array}{l}\text { Case } \\
\text { Sample }\end{array}$ & year & $\begin{array}{c}\text { \#blocks/ } \\
\text { slides }\end{array}$ & DNA yield( $\boldsymbol{\mu g})$ & $\begin{array}{l}\text { \% SNP } \\
\text { genotype }\end{array}$ \\
\hline 423197 & 1969 & 3 & $\begin{array}{c}\text { *no normal } \text { tissue } \\
\text { NA }\end{array}$ & NA \\
525593 & 1984 & 14 & 48 & .891 \\
620691 & 1985 & 3 & 6 & .971 \\
723932 & 1990 & 8 & 12 & .989 \\
826190 & 1992 & $1 / 1$ & 5 & .873 \\
\hline
\end{tabular}

FFPE tissue block and stained with hematoxylin and eosin (H\&E) for histological review. Tumor rich areas were circled on the H\&E slide and aligned with the original tissue block; full face cuts $(4 \times 10$ micron $)$ were made from blocks containing no tumor tissue to capture grossly uninvolved (i.e. "normal") tissue. There was sufficient normal tissue in 4 of these 5 samples; the oldest sample did not have sufficient normal tissue for extraction (case 423197 in Table 1).

Tissue punches from normal tissue were combined and de-paraffinized in Citri Solv followed by dehydration in $100 \%$ Ethanol. DNA was isolated from the dried tissue using the Qiagen QIAamp DNA FFPE Tissue Kit. The yield, purity and integrity of DNA samples was assessed using spectrophotometry and gel electrophoresis. Both yield and purity was excellent, with at least $5 \mu \mathrm{g}$ obtained from each sample and A260/280 ratios of 1.8. However, the integrity of the DNA was questionable, with significant fragmentation detectable upon electrophoresis (data not shown).

We provided DNA samples for 4 cases with normal tissue extracted to deCODE Genetics for genome-wide genotyping with the $610 \mathrm{Q}$ platform. For one of these 4 cases (723932), we also provided deCODE with a DNA sample which was extracted from whole blood. For another case with an FFPE extracted sample (826190) we had previously received GW genotyping from deCODE for a DNA sample extracted from whole blood. For these two-paired samples of DNA from FFPE normal and DNA from whole blood from the same individuals we estimated reliability. This research was approved by the University of Utah IRB, and all subjects were appropriately consented.

\section{Results and Discussion}

Table 1 summarizes the 5 tissue samples considered (the first of which yielded no normal DNA). The genotyping call rates for the 4 DNA case samples from FFPE ranged from 0.873 to 0.989 . For the case for which we submitted both DNA from whole blood and also DNA from normal tissue from FFPE (marker yield $=0.999)$ at the same time (723932), we observed 0.9998 reproducibility in genotyping calls. For one of the other cases with an FFPE sample (826190) we had obtained GW genotyping results previously; concordance between the 2 samples was 0.993 . The only slightly less than $100 \%$ concordance may be due to drop outs or may represent the very low genotyping error rates observed for SNP arrays; such a low error rate is likely to have little or no impact on identity-by descent inference for disease linkage. These surprisingly high success rates for number of calls and for reproducibility of calls for DNA extracted from FFPE may be the norm, rather than the exception, as these 4 samples were chosen randomly and represent a range of FFPE tissue block quality, quantity and age.

Previous studies have illustrated that DNA present within FFPE tissue blocks can be used for molecular studies [2-7]. However, these previous studies have primarily focused on CNV LOH studies, and small numbers of SNPs; they did not perform or compare genome-wide genotyping results for normal tissue,. There is significant variability in how FFPE samples are collected, processed, and stored [8]. Factors that affect the quality of nucleic acids within FFPE tissue blocks include the time from de-vascularization to fixation, the method of fixation, and the age of the tissue block [8]. While pre-analytical testing can provide some information about the suitability of a sample for downstream molecular applications ([9], it is often unknown whether a given technology can yield useful data from a sample until the final analyses.

\section{Conclusions}

Our attempt to generate genome-wide genotyping data for individuals not available for genotyping, has been both simple and revolutionary in several ways. We have shown that "normal" tissue from archived FFPE samples (even decades old) is an excellent source of germline DNA for individuals no longer available for sampling. We have also shown that both high quality and high yield genome-wide genotyping data can be obtained from DNA extracted from normal FFPE tissue (even when samples are stored long term). Finally, we propose that the GW genotyping data from DNA extracted from normal tissue from FFPE samples is more than adequate to allow GW linkage analysis despite significant fragmentation, and might be considered adequate for GW association studies (with some modifications to methods, given the slightly smaller number of SNPs available).

In Utah, as an example, the largest health care provider has wisely stored all pathology samples available since the 1960s. For such long-standing bio-repositories, the methods we propose could be important for the study of disorders which are no longer commonly diagnosed worldwide, but which still have important lessons to teach, and for which stored samples are available, e.g. pandemic influenza. These findings are especially important for disorders for which there are few living cases (e.g. disorders with short survival times). 
We are aware that some companies have developed additional protocols designed to improve yield of genotyping data from DNA extracted from FFPE samples; this will further enhance the value of stored FFPE samples. Our innovative solution to procuring genome-wide genotyping data for informative individuals not otherwise available for sampling has provided a powerful way forward for a number of different genetic studies which require genome-wide genotyping data of good quality and quantity. Those researchers and facilities with the foresight to store FFPE tissue have at their fingertips a vastly informative and valuable resource that we propose will allow many investigations not previously thought possible.

\section{List of Abbreviations}

FFPE: formalin fixed paraffin embedded; SNPs: single nucleotide polymorphism.

\section{Acknowledgements and Funding}

L-C-A acknowledges support from the National Library of Medicine grant LM009331, and a subcontract from Johns Hopkins University with funds provided by grant R01 CA89600 from the NIH National Cancer Institute. Supported in part by the NCl Cancer Center Support Grant P30 CA42014-19. We acknowledge the assistance of Jeanette Rasmussen and Paulette Bowman at the TRAC facility at the Huntsman Cancer Center. This paper is subject to the NIH Public Access Policy.

\section{Author details}

${ }^{1}$ Genetic Epidemiology, Department of Internal Medicine, University of Utah School of Medicine, Salt Lake City, UT, USA. ${ }^{2}$ George E. Wahlen Department of Veterans Affairs Medical Center, Salt Lake City, Utah, USA. ${ }^{3}$ Tissue Resource and Applications Core, Huntsman Cancer Institute, University of Utah, Salt Lake City, UT, USA. ${ }^{4}$ Department of Pathology, University of Utah Health Sciences Center, Salt Lake City, UT, USA. ${ }^{5}$ The ARUP Institute for Clinical and Experimental Pathology, SLC, Utah, USA.

\section{Authors' contributions}

The project was conceived of by LACA who also provided tissue; PB performed the pathologic examination and selection of sample tissue; KC performed the DNA extraction under the direction of AG; all authors contributed to study design and manuscript revisions. All authors read and approved the final manuscript.

\section{Competing interests}

The authors declare that they have no competing interests.

Received: 4 March 2011 Accepted: 26 May 2011 Published: 26 May 2011

\section{References}

1. Allen-Brady K, Norton PA, Farnham JM, Teerlink C, Cannon-Albright LA: Significant Linkage Evidence for a Predisposition Gene for Pelvic Floor Disorders on Chromosome 9q21. Am J Hum Genet 2009, 84(5):678-82.

2. Gnanapragasam VJ: Unlocking the molecular archive: the emerging use of formalin-fixed paraffin-embeddeed tissue for biomarker research in urological cancer. Br J Urol International 2009, , 105: 274-278.

3. Oosting J, Lips EH, van Ejik R, Eilers PH, Szubhai K, Wijmenga C, Morreau H, van Wezel T: High-resolution copy number analysis of paraffinembedded archival tissue using SNP BeadArrays. Genome Res 2007, 17(3):368-76, Epub 2007 Jan 31

4. Hennig G, Gehrmann M, Stropp U, Brauch H, Fritz P, Eichelbaum M, Schwab M, Schroth W: Automated extraction of DNA and RNA from a single formalin-fixed paraffin-embedded tissue section for analysis of both single-nucleotide polymorphisms and mRNA expression. Clin Chem 2010, 56(12):1845-53.
5. Sikora M, Thibert JN, Salter J, Dowsett M, Johnson MD, Rae JM: Highefficiency genotype analysis from formalin-fixed paraffin-embedded tumor tissues. Pharmacogenomics J 2010.

6. Thompson ER, Herbertt SC, Forrest SM, Camplbell IG: Whole genome SNP arrays using DNA derived from formalin-fixed, paraffin-embedded ovarian tumor tissue. Hum Mutat 2005, 26(4):384-9.

7. Reid AH, Fanning TG, Hultin JV, Taubenberger JK: Origin and evolution of the 1918 "Spanish" influenza virus hemagglutinin gene. Proc Natl Acad Sci 1999, 96:1651-56.

8. Hewitt SM, Lewis FA, Yanxiang C, Conrad RC, Cronin M, Danenberg KD, Goralski TJ, Langmore JP, et al: Tissue Handling and Specimen Preparation in Surgical Pathology: Issues Concerning the Recovery of Nucleic Acids from Formalin-Fixed, Paraffin-Embedded Tissue. Arch Pathol Lab Med 2008, 132:1929-1936.

9. Panaro NJ, Yuen PK, Sakazume T, Fortina P, Kricka LJ, Wilding P: Evaluation of DNA fragment sizing and quantification by the agilent 2100 bioanalyzer. Clin Chem 2000, 46:1851-1853.

doi:10.1186/1756-0500-4-159

Cite this article as: Cannon-Albright et al:: High quality and quantity Genome-wide germline genotypes from FFPE normal tissue. BMC Research Notes 2011 4:159.

\section{Submit your next manuscript to BioMed Central and take full advantage of:}

- Convenient online submission

- Thorough peer review

- No space constraints or color figure charges

- Immediate publication on acceptance

- Inclusion in PubMed, CAS, Scopus and Google Scholar

- Research which is freely available for redistribution 